\title{
UPPER BOUNDS ON CERTAIN FUNCTIONALS DEFINED ON GROUPS OF LINEAR OPERATORS*
}

\author{
MAREK NIEZGODA ${ }^{\dagger}$
}

\begin{abstract}
The problem of estimating certain functionals defined on a group of linear operators generating a group induced cone (GIC) ordering is studied. A result of Berman and Plemmons [Math. Inequal. Appl., 2(1):149-152, 1998] is extended from the sum function to Schur-convex functions. It is shown that the problem has a closed connection with both Schur type inequality and weak group majorization. Some applications are given for matrices.
\end{abstract}

Key words. Group majorization, GIC ordering, Normal decomposition system, Cone preordering, Schur type inequality, Schur-convex function, Eigenvalues, Singular values.

AMS subject classifications. 06F20, 39B62, 15A48, $15 \mathrm{~A} 18$.

1. Introduction. Berman and Plemmons [2] proved that the functional

$$
f(U)=\sum_{i=1}^{n} \max _{j}\left\{\left(U^{T} M_{j} U\right)_{i i}\right\}
$$

over all $n \times n$ orthogonal matrices $U$ is maximized by an orthogonal matrix $Q$ which simultaneously diagonalizes the symmetric matrices $M_{j}, j=1, \ldots, k$. An analogous result holds for Hermitian matrices [2, Section 3].

In the present paper we study a similar problem for a general linear space endowed with the structure of normal decomposition (ND) system (to be defined below). Also, we replace the sum function in (1.1) by an increasing function with respect to certain vector orderings (see Section 2). Some applications are given for matrices in Section 3. A further extension to weak group majorization is discussed in Section 4.

2. Results. Let $V$ be a finite-dimensional real linear space equipped with an inner product $\langle\cdot, \cdot\rangle$. By $O(V)$ we denote the orthogonal group acting on $V$. Let $G$ be a closed subgroup of $O(V)$. The group majorization induced by $G$, abbreviated as $G$-majorization and written as $\preceq_{G}$, is the preordering on $V$ defined by

$$
y \preceq_{G} x \text { iff } y \in \operatorname{conv} G x,
$$

where conv $G x$ denotes the convex hull of the orbit $G x:=\{g x: g \in G\}$ (see [18]).

Let $(\cdot)_{\downarrow}: V \rightarrow V$ be a $G$-invariant map, that is $(g x)_{\downarrow}=x_{\downarrow}$ for any $x \in V$ and $g \in G$. We say that $\left(V, G,(\cdot)_{\downarrow}\right)$ is a normal decomposition (ND) system (see $[10,11]$ ) if

(A1) for any $x \in V$ there exists $g \in G$ satisfying $x=g x_{\downarrow}$,

(A2) $\max _{g \in G}\langle x, g y\rangle=\left\langle x_{\downarrow}, y_{\downarrow}\right\rangle$ for all $x, y \in V$.

\footnotetext{
*Received by the editors 27 November 2005. Accepted for publication 13 June 2006. Handling Editor: Shmuel Friedland.

$\dagger$ Department of Applied Mathematics, Agricultural University of Lublin, P.O. Box 158, Akademicka 13, PL-20-950 Lublin, Poland (marek.niezgoda@ar.lublin.pl).
} 
In this event, it can be deduced (see [10, Thm 2.4]) that the set $D=V_{\downarrow}$, the range of $(\cdot)_{\downarrow}$, is a closed convex cone. Under axioms (A1)-(A2), the group majorization $\preceq_{G}$ is said to be a group induced cone $\left(\right.$ GIC) ordering $[4,5,6,18]$. The operator $(\cdot)_{\downarrow}$ is called a normal map. See $[4,5,6,10,11]$ for examples of ND systems and GIC orderings. See also Section 3.

Axiom (A1) generalizes the Spectral Theorem for Hermitian (or real symmetric) matrices as well as the Singular Value Decomposition Theorem for complex (or real) matrices [4]. On the other hand, (A2) can be viewed as an extension of the fundamental von Neumann trace result on singular values [4] and of the analogous result of Miranda and Thompson $[14,15]$. This makes it possible to apply results for a general ND system in matrix theory, convex analysis, optimization, etc.

The notion of ND system was introduced by Lewis (see [10, pp. 928-929] and [11, pp. 817-818]). The corresponding theory of group induced cone orderings was developed by Eaton and Perlman [7], Eaton [4, 5, 6], Giovagnoli and Wynn [8], Steerneman [18] and Niezgoda [16, 17].

For an ND system $\left(V, G,(\cdot)_{\downarrow}\right)$ with $D$ being the range of $(\cdot)_{\downarrow}$, it can be shown that $x_{\downarrow}$ is the unique element of the set $D \cap G x$. Denote

$$
W:=\operatorname{span} D \text { and } H:=\left\{h=g_{\mid W}: g \in G, g W=W\right\} .
$$

It is known (see [16, Thm 3.2], [16, p. 14], [18, Thm 4.1] and [9]) that the following statements (i)-(iv) are mutually equivalent:

(i) The group majorizations $\preceq_{G}$ and $\preceq_{H}$ are equivalent on $W$, that is

$$
y \preceq_{G} x \text { iff } y \preceq_{H} x \text { for all } x, y \in W \text {. }
$$

(ii) $\left(W, H,(\cdot)_{\downarrow}\right)$ is an ND system (with the inherited normal map).

(iii) Schur type inequality holds, that is

$$
P x \preceq_{H} x_{\downarrow} \text { for all } x \in V,
$$

where $P$ is the orthogonal projection from the space $V$ onto the space $W$.

(iv) $H$ is a finite reflection group.

Let $C \subset W$ be a convex cone. The cone preordering $\preceq_{C}$ induced by $C$ is defined as follows:

$$
y \preceq_{C} x \text { iff } x-y \in C
$$

for $x, y \in W$. A function $\varphi: W \rightarrow \mathbb{R}$ is said to be $C$-increasing (resp. $H$-increasing) if $y \preceq_{C} x$ (resp. $y \preceq_{H} x$ ) implies $\varphi(y) \leq \varphi(x)$ for $x, y \in W$. The function $\varphi$ is said to be $C H$-increasing if it is both $C$-increasing and $H$-increasing. We say that $C$ has max property if for any vectors $a_{1}, \ldots, a_{k} \in W$ there exists a maximal vector max $a_{j}$ with respect to $\preceq_{C}$. We call a linear operator $L: V \rightarrow V C$-positive if $L C \subset C$. Observe that the $C$-positivity of $L$ implies $L y \preceq_{C} L x$ whenever $y \preceq_{C} x$.

TheOREm 2.1. Let $\left(V, G,(\cdot)_{\downarrow}\right)$ be an ND system satisfying any of the above equivalent conditions (i)-(iv). Assume $C \subset W$ is an $H$-invariant convex cone. Let $\varphi: W \rightarrow \mathbb{R}$ be a $C H$-increasing function. 
(I) Let $w, w_{1}, \ldots, w_{k}$ be vectors in $W$. If $w_{j} \preceq_{C} w$ for $j=1, \ldots, k$, then for each $g \in G$

$$
\begin{gathered}
P g w_{j} \preceq_{C} P g w \preceq_{H} w \quad \text { for } j=1, \ldots, k, \\
\max _{j} \varphi\left(P g w_{j}\right) \leq \varphi(P g w) \leq \varphi(w) .
\end{gathered}
$$

(II) If, in addition, $C$ has max property, then for each $g \in G$

$$
\begin{gathered}
\max _{j} P g w_{j} \preceq_{C} P g w \preceq_{H} w, \\
\varphi\left(\max _{j} P g w_{j}\right) \leq \varphi(P g w) \leq \varphi(w) .
\end{gathered}
$$

(III) In particular, if $C$ has max property and $w=\max _{j} w_{j}$, then for each $g \in G$

$$
\varphi\left(\max _{j} P g w_{j}\right) \leq \varphi\left(\max _{j} w_{j}\right),
$$

i.e., the functional

$$
f(g):=\varphi\left(\max _{j} P g w_{j}\right), \quad g \in G,
$$

is maximized by $g=i d$, the identity operator on $V$.

Proof. Fix arbitrarily $g \in G$. We shall prove that the linear operator $P g$ is $C$ positive. Denote $D=V_{\downarrow}$. Recall that $\left\{x_{\downarrow}\right\}=D \cap G x$ for $x \in V$. Using (2.1) and the $G$-invariance of the normal map $(\cdot)_{\downarrow}: V \rightarrow D$, we obtain $P g z \preceq_{H}(g z)_{\downarrow}=z_{\downarrow}$ for $z \in W$.

On the other hand, employing condition (A1) for the ND systems $\left(V, G,(\cdot)_{\downarrow}\right)$ and $\left(W, H,(\cdot)_{\downarrow}\right)$, for each $z \in W$ we get $z=g_{0} z_{\downarrow}$ and $z=h_{0} d$ for some $g_{0} \in G$, $h_{0} \in H$ and $d \in D$. By [16, Lemma 2.1], we derive $d=z_{\downarrow}$. Therefore $z=h_{0} z_{\downarrow}$. In consequence, $\mathrm{Hz}=H z_{\downarrow}$ for $z \in W$. It now follows that $\mathrm{Pgz} \in \operatorname{conv} H z_{\downarrow}=\operatorname{conv} \mathrm{Hz}$, since Pgz $\preceq_{H} z_{\downarrow}$. Hence

$$
\operatorname{Pg} z \preceq_{H} z \text { for } z \in W \text {. }
$$

Since $C$ is $H$-invariant, we obtain $H z \in C$ and conv $H z \subset C$ for $z \in C$. Therefore $P g z \in C$ for $z \in C$, since $P g z \in \operatorname{conv} H z$ by (2.8). This yields the $C$-positivity of $\mathrm{Pg}$, as required.

(I). Since $w_{j} \preceq_{C} w$ for $j=1, \ldots, k$, we get $P g w_{j} \preceq_{C} P g w$ by the $C$-positivity of $P g$. Additionally, $P g w \preceq{ }_{H} w$ by (2.8). This completes the proof of (2.2).

Applying (2.2) and the fact that $\varphi$ is $C H$-increasing, one obtains $\varphi\left(P g w_{j}\right) \leq$ $\varphi(P g w) \leq \varphi(w)$ for $j=1, \ldots, k$. This proves (2.3).

(II). Suppose that $C$ has max property. Then there exists $\max _{j} P g w_{j}$. Using (2.2) we derive (2.4). Moreover, (2.4) implies (2.5), because $\varphi$ is $C H$-increasing. 
(III). Substituting $w:=\max _{j} w_{j}$ into (2.5) yields (2.6). Since $w_{j} \in W$ and $P w_{j}=w_{j}$, (2.6) means that the functional defined by (2.7) is maximized by the identity operator $i d$ on $V$.

Corollary 2.2. Let $\left(V, G,(\cdot)_{\downarrow}\right)$ be an ND system satisfying any of the equivalent conditions (i)-(iv). Assume $C \subset W$ is an $H$-invariant convex cone having max property. Suppose that $\varphi: W \rightarrow \mathbb{R}$ is a $C H$-increasing function.

Let $v_{1}, \ldots, v_{k}$ be vectors in $V$ such that $g_{0} v_{1}, \ldots, g_{0} v_{k} \in W$ for some $g_{0} \in G$. Then $g_{0}$ maximizes the functional

$$
f(g):=\varphi\left(\max _{j} P g v_{j}\right), \quad g \in G .
$$

Proof. Apply Theorem 2.1, part (III), for $w_{j}:=g_{0} v_{j} \in W, j=1, \ldots, k$, and $w:=\max _{j} g_{0} v j$.

Theorem 2.1, part (I), can be modified. See Theorem 3.2 for application of the result below.

COROLlary 2.3. Under the hypotheses of Theorem 2.1, assume that $\varphi$ is $\mathrm{H}$ increasing on some set $W_{0}$ (in place of $W$ ) such that $W_{\downarrow} \subset W_{0} \subset W$. Suppose that there exists a subgroup $H_{0} \subset H$ satisfying (i) for each $h_{0} \in H_{0}$ there exists $g_{0} \in G$ such that $h_{0} P=P g_{0}$, and (ii) for each $w \in W$ there exists an $h_{0} \in H_{0}$ such that $h_{0} w \in W_{0}$.

Then

$$
\max _{j} \varphi\left(P g_{0} g w_{j}\right) \leq \varphi\left(P g_{0} g w\right) \leq \varphi\left(w_{\downarrow}\right),
$$

where $h_{0} P g w=P g_{0} g w \in W_{0}$.

Proof. Applying (i)-(ii) we take an $h_{0} \in H_{0}$ and $g_{0} \in G$ such that $h_{0} P g w \in$ $W_{0}$ and $h_{0} P=P g_{0}$. Since $C$ is $H$-invariant, $H_{0} \subset H$ and $\preceq_{H}$ is $H$-invariant, it follows from (2.3) that $h_{0} P g w_{j} \preceq_{C} h_{0} P g w$ and $h_{0} P g w \preceq_{H} w_{\downarrow}$ with $w_{\downarrow} \in W_{\downarrow} \subset W_{0}$. Therefore (2.9) holds.

3. Applications. In this section we interpret the results of Section 2 in matrix setting. We consider two special cases. The first leads to a result generalizing a theorem of Berman and Plemmons [2] (see Corollary 3.1).

To do this, we set

$V:=$ the linear space $\mathbb{S}_{n}$ of $n \times n$ real symmetric matrices,

$G:=$ the group of operators of the form $X \rightarrow U X U^{T}, X \in V$, where $U$ runs over the group $\mathbb{O}_{n}$ of $n \times n$ orthogonal matrices,

$X_{\downarrow}:=\operatorname{diag} \lambda(X)$ for $X \in \mathbb{S}_{n}$, where $\lambda(X)$ denotes the vector of the eigenvalues of $X$ arranged in decreasing order on the main diagonal, and

$\operatorname{diag} x$ stands for the diagonal matrix with $x \in \mathbb{R}^{n}$. We adopt the convention that the members of $\mathbb{R}^{n}$ are row $n$-vectors. Then $\left(V, G,(\cdot)_{\downarrow}\right)$ is an ND system by virtue of the Spectral Theorem and Theobald's trace inequality on eigenvalues (see $[4,12,19])$. 
Also, it is well known that $D=V_{\downarrow}$ is the convex cone of $n \times n$ real diagonal matrices with decreasingly ordered diagonal entries.

In addition, $\left(W, H,(\cdot)_{\downarrow}\right)$ is an ND system for

$W:=$ the linear space of $n \times n$ diagonal matrices,

$H:=$ the group of operators of the form $X \rightarrow U X U^{T}, X \in W$, where $U$ varies over

the group $\mathbb{P}_{n}$ of $n \times n$ permutation matrices.

The orthoprojector $P$ from $V$ onto $W$ is given by

$$
P(X)=\operatorname{diag} \Delta(X) \text { for } X \in \mathbb{S}_{n},
$$

where the symbol $\Delta(\cdot)$ means "the diagonal of".

Conditions (i)-(iv) of Section 2 are fulfilled (see [16, Example 4.1] for details). In particular, inequality (2.1) takes the form of the classical Schur inequality:

$$
\Delta\left(U X U^{T}\right) \preceq_{m} \lambda(X) \text { for } X \in S_{n} \text { and } U \in O_{n} .
$$

Here the relation $\preceq_{m}$ is the ordinary majorization on $\mathbb{R}^{n}$ defined as follows (see [13, p. 7]). Given two vectors $a, b \in \mathbb{R}^{n}$, we say that $a$ majorizes $b$ if

$$
\sum_{i=1}^{m} b_{[i]} \leq \sum_{i=1}^{m} a_{[i]} \text { for } m=1, \ldots, n,
$$

with equality for $m=n$. By $c_{[i]}$ we denote the $i$ th largest entry of a vector $c=$ $\left(c_{1}, \ldots, c_{n}\right) \in \mathbb{R}^{n}$. A function $\psi: \mathbb{R}^{n} \rightarrow \mathbb{R}$ is said to be Schur-convex if $\psi(b) \leq \psi(a)$ whenever $b \preceq_{m} a$ for $a, b \in \mathbb{R}^{n}$ (cf. [13, p. 54]).

It is known that $\preceq_{m}$ is the group majorization on $\mathbb{R}^{n}$ induced by the permutation group $\mathbb{P}_{n}$ (see [4, p. 16]). There is a closed connection between the $H$-majorization $\preceq_{H}$ on $W$ and the ordinary majorization $\preceq_{m}$ on $\mathbb{R}^{n}$. Namely,

$$
\operatorname{diag} y \preceq_{H} \operatorname{diag} x \quad \text { iff } \quad y \preceq_{m} x \quad \text { for } x, y \in \mathbb{R}^{n} .
$$

To see this, employ the formula

$$
U(\operatorname{diag} x) U^{T}=\operatorname{diag}\left(x U^{T}\right) \text { for } U \in \mathbb{P}_{n} \text { and } x \in \mathbb{R}^{n} .
$$

Applying Corollary 2.2 for

$C:=$ the convex cone of $n \times n$ diagonal matrices with nonnegative diagonal entries, $\preceq_{C}:=$ the entrywise ordering on $W$,

$\max :=$ the maximum operator with respect to the entrywise ordering $\preceq_{C}$ on $W$ ， we get

Corollary 3.1. Let $M_{j}, j=1, \ldots, k$, be a collection of pairwise commuting $n \times n$ symmetric matrices, and let $U_{0}$ be an $n \times n$ orthogonal matrix such that the similarity operator $g_{0}:=U_{0}(\cdot) U_{0}^{T}$ simultaneously diagonalizes the $M_{j}$. 
Let $\psi: \mathbb{R}^{n} \rightarrow \mathbb{R}$ be an $\mathbb{R}_{+}^{n}$-increasing function. If $\psi$ is Schur-convex then $U_{0}$ maximizes the functional

$$
f(U):=\psi\left(\max _{j} \Delta\left(U M_{j} U^{T}\right)\right)
$$

over all $n \times n$ orthogonal matrices $U$.

Proof. Apply (3.1)-(3.5) and Corollary 2.2 for the function $\varphi(X):=\psi(x)$, where $X:=\operatorname{diag} x$ for $x \in \mathbb{R}^{n}$.

The above result extends the mentioned theorem of Berman and Plemmons [2]. To see this, use Corollary 3.1 for the function $\psi(x):=\sum_{i=1}^{n} x_{i}$ for $x=\left(x_{1}, \ldots, x_{n}\right) \in \mathbb{R}^{n}$.

We are now interested in using Theorem 2.1 and Corollary 2.3 to obtain a result for singular values of matrices in $\mathbb{M}_{n}$ (see Theorem 3.2) under the action of the group $G_{0}$ of orthogonal equivalences. However, there are no $G_{0}$-invariant convex cones in $\mathbb{M}_{n}$ (except $\mathbb{M}_{n}$ and $\{0\}$ ). To avoid this difficulty, we use embedding of the space $\mathbb{M}_{n}$ in $\mathbb{M}_{2 n}$. Namely, let $V$ be the linear space of all $2 n \times 2 n$ matrices of the form

$$
\left[\begin{array}{cc}
\alpha I & X \\
X^{T} & \alpha I
\end{array}\right]
$$

where $X$ is an $n \times n$ real matrix, $I$ is the $n \times n$ identity matrix and $\alpha$ is a real number. Put $G$ to be the group of all linear maps $g$ from $V$ to $V$ of the form

$$
\begin{aligned}
{\left[\begin{array}{cc}
\alpha I & X \\
X^{T} & \alpha I
\end{array}\right] } & \rightarrow\left[\begin{array}{cc}
U_{1} & 0 \\
0 & U_{2}
\end{array}\right]\left[\begin{array}{cc}
\alpha I & X \\
X^{T} & \alpha I
\end{array}\right]\left[\begin{array}{cc}
U_{1} & 0 \\
0 & U_{2}
\end{array}\right]^{T} \\
& =\left[\begin{array}{cc}
\alpha I & U_{1} X U_{2}^{T} \\
\left(U_{1} X U_{2}^{T}\right)^{T} & \alpha I
\end{array}\right],
\end{aligned}
$$

where $U_{1}$ and $U_{2}$ run over the group of $n \times n$ orthogonal matrices. Then the Singular Value Decomposition Theorem and the von Neumann trace inequality for $n \times n$ matrices imply that $\left(V, G,(\cdot)_{\downarrow}\right)$ is an ND system for the normal map $(\cdot)_{\downarrow}$ defined on $V$ by

$$
\left[\begin{array}{cc}
\alpha I & X \\
X^{T} & \alpha I
\end{array}\right]_{\downarrow}:=\left[\begin{array}{cc}
\alpha I & \operatorname{diag} s(X) \\
\operatorname{diag} s(X) & \alpha I
\end{array}\right]
$$

where $s(X):=\left(s_{1}(X), \ldots, s_{n}(X)\right)$ is the $n$-vector of the singular values of $X$ arranged in decreasing order (cf. [1, p. 106], [4, pp. 17-18]). That is, the numbers $s_{1}(X) \geq \ldots \geq$ $s_{n}(X)$ are the nonnegative square roots of the eigenvalues of the positive semi-definite matrix $X^{T} X$. The range of the normal map $(\cdot)_{\downarrow}$ is the convex cone

$$
D=\left\{\left[\begin{array}{cc}
\alpha I & \operatorname{diag} x \\
\operatorname{diag} x & \alpha I
\end{array}\right]: \alpha \in \mathbb{R}, x \in \mathbb{R}_{+\downarrow}^{n}\right\},
$$

where $\mathbb{R}_{+\downarrow}^{n}$ is the set of nonnegative real $n$-vectors with decreasingly ordered entries.

The system $\left(W, H,(\cdot)_{\downarrow}\right)$ is given by the space

$$
W:=\left\{\left[\begin{array}{cc}
\alpha I & \operatorname{diag} x \\
\operatorname{diag} x & \alpha I
\end{array}\right]: \alpha \in \mathbb{R}, x \in \mathbb{R}^{n}\right\},
$$


and by the group $H$ of linear operators from $W$ to $W$ of the type

$$
\left[\begin{array}{cc}
\alpha I & \operatorname{diag} x \\
\operatorname{diag} x & \alpha I
\end{array}\right] \rightarrow\left[\begin{array}{cc}
\alpha I & U_{1} \operatorname{diag} x U_{2}^{T} \\
U_{1} \operatorname{diag} x U_{2}^{T} & \alpha I
\end{array}\right],
$$

where $U_{1}$ and $U_{2}$ run over the group of $n \times n$ generalized permutation matrices (i.e., matrices with exactly one nonzero entry \pm 1 in each column and each row) such that

$$
U_{1} \operatorname{diag} x U_{2}^{T}=\operatorname{diag}\left( \pm x_{i_{1}}, \ldots, \pm x_{i_{n}}\right)
$$

for any choice of \pm signs and of permutation $x_{i_{1}}, \ldots, x_{i_{n}}$ of the entries of $x$. Therefore

$$
\left[\begin{array}{cc}
\beta I & \operatorname{diag} y \\
\operatorname{diag} y & \beta I
\end{array}\right] \preceq_{H}\left[\begin{array}{cc}
\alpha I & \operatorname{diag} x \\
\operatorname{diag} x & \alpha I
\end{array}\right] \quad \text { iff } \beta=\alpha \text { and } y \preceq_{a w} x .
$$

Here the relation $y \preceq$ aw $x$ means that $(3.3)$ holds for $a:=\left(\left|x_{1}\right|, \ldots,\left|x_{n}\right|\right)$ and $b:=$ $\left(\left|y_{1}\right|, \ldots,\left|y_{n}\right|\right)$ (cf. [4, p. 16]). Observe that

$$
\begin{aligned}
y \preceq_{a w} x & \text { iff }\left(|y|_{\downarrow},-|y|_{\uparrow}\right) \preceq_{m} \quad\left(|x|_{\downarrow},-|x|_{\uparrow}\right) \\
& \text { iff }\left(\alpha 1_{n}+|y|, \alpha 1_{n}-|y|\right) \preceq_{m}\left(\alpha 1_{n}+|x|, \alpha 1_{n}-|x|\right)
\end{aligned}
$$

(cf. [1, p. 107]), where $|x|$ denotes the vector of the moduli of the entries of $x$, and the vector $|x|_{\downarrow}$ (resp. $|x|_{\uparrow}$ ) consists of the entries of $|x|$ arranged decreasingly (resp. increasingly).

The orthoprojector $P$ from $V$ onto $W$ is given by

$$
P\left[\begin{array}{cc}
\alpha I & X \\
X^{T} & \alpha I
\end{array}\right]=\left[\begin{array}{cc}
\alpha I & \operatorname{diag} \Delta(X) \\
\operatorname{diag} \Delta(X) & \alpha I
\end{array}\right],
$$

where $\Delta(X)$ stands for the diagonal of $X$.

Denote by $\mathbb{L}_{2 n}$ the Loewner cone of all $2 n \times 2 n$ positive semidefinite matrices. Notice that $\mathbb{L}_{2 n}$ is $G$-invariant. Define

$$
C:=\mathbb{L}_{2 n} \cap W .
$$

Evidently, $C$ is an $H$-invariant convex cone. In addition, $\preceq_{C}$ is the cone preordering on $W$ such that

$$
\begin{gathered}
{\left[\begin{array}{cc}
\beta I & \operatorname{diag} y \\
\operatorname{diag} y & \beta I
\end{array}\right] \underset{\text { implies }}{\preceq_{C}}\left[\begin{array}{cc}
\alpha I & \operatorname{diag} x \\
\operatorname{diag} x & \alpha I
\end{array}\right]} \\
\left(\beta 1_{n}+y, \beta 1_{n}-y\right)_{\downarrow} \leq \quad\left(\alpha 1_{n}+x, \alpha 1_{n}-x\right)_{\downarrow},
\end{gathered}
$$

where $\leq$ is the entrywise ordering on $\mathbb{R}^{2 n}$ and the $n$-vector $1_{n}$ consists of ones. To see this, note that the vectors in inequality (3.10) consist of the eigenvalues of the matrices in (3.9), respectively, (see [1, p. 105]), and use Weyl's monotonicity theorem [3, Cor. III.2.3, p. 63]. 
Take $H_{0}$ to be the subgroup of $H$ consisting of all operators of the form $h_{0}=$ $U_{0}(\cdot) I$, where $U_{0}=\operatorname{diag}( \pm 1, \ldots, \pm 1)$ is a sign change matrix.

With the above notation, and from Theorem 2.1 and Corollary 2.3, we obtain

TheOrem 3.2. Let $\alpha_{j}$ and $M_{j}$ for $j=0,1, \ldots, k$ be, respectively, real numbers and $n \times n$ real matrices, and let

$$
\widetilde{M}_{j}:=\left[\begin{array}{cc}
\alpha_{j} I & M_{j} \\
M_{j}^{T} & \alpha_{j} I
\end{array}\right]
$$

be the corresponding matrices in $V$ such that $\widetilde{M}_{j} \preceq_{\mathbb{L}_{2 n}} \widetilde{M}_{0}$. Assume that there exists an operator $g_{0}$ in $G$ simultaneously sending the $\widetilde{M}_{j}$ into $W$, that is

$$
g_{0} \widetilde{M}_{j}=\left[\begin{array}{cc}
\alpha_{j} I & \operatorname{diag} \sigma\left(M_{j}\right) \\
\operatorname{diag} \sigma\left(M_{j}\right) & \alpha_{j} I
\end{array}\right] \quad \text { for } j=0,1, \ldots, k,
$$

where $\sigma\left(M_{j}\right):=\left( \pm s_{i_{1}}\left(M_{j}\right), \ldots, \pm s_{i_{n}}\left(M_{j}\right)\right)$ and $s_{1}\left(M_{j}\right) \geq \ldots \geq s_{n}\left(M_{j}\right) \geq 0$ are the singular values of $M_{j}$ with any choice of \pm signs and any permutation $i_{1}, \ldots, i_{n}$ of $1, \ldots, n$.

Let $\psi$ be a real function which is Schur-convex and entrywise increasing on $\mathbb{R}^{2 n}$. Then

$$
\begin{gathered}
\max _{1 \leq j \leq k} \psi\left(\alpha_{j} 1_{n}+\Delta\left(U_{1} M_{j} U_{2}^{T}\right) U_{0}, \alpha_{j} 1_{n}-\Delta\left(U_{1} M_{j} U_{2}^{T}\right) U_{0}\right) \\
\leq \psi\left(\alpha_{0} 1_{n}+s\left(M_{0}\right), \alpha_{0} 1_{n}-s\left(M_{0}\right)\right)
\end{gathered}
$$

for any orthogonal $n \times n$ matrices $U_{1}$ and $U_{2}$, and for some $U_{0}=\operatorname{diag}( \pm 1, \ldots, \pm 1)$ such that $\Delta\left(U_{1} M_{0} U_{2}^{T}\right) U_{0}=\left|\Delta\left(U_{1} M_{0} U_{2}^{T}\right)\right|$.

Proof. Consider the functions

$$
\varphi(X):=\psi\left(\alpha 1_{n}+x, \alpha 1_{n}-x\right) \text { and } \widetilde{\varphi}(X):=\psi\left(\alpha 1_{n}+|x|, \alpha 1_{n}-|x|\right)
$$

for $X=\left[\begin{array}{cc}\alpha I & \operatorname{diag} x \\ \operatorname{diag} x & \alpha I\end{array}\right]$ with $x \in \mathbb{R}^{n}$ and $\alpha \in \mathbb{R}$. It is obvious that $\varphi(X)=\widetilde{\varphi}(X)$ for nonnegative $x$.

It follows that $\varphi$ is $C$-increasing, because (3.9)-(3.10) are met, and $\psi$ is Schurconvex, permutation-invariant and entrywise increasing. Using (3.6) and (3.12) we obtain that $\widetilde{\varphi}$ is $H$-invariant. Applying (3.7)-(3.8) and (3.12) we deduce that $\widetilde{\varphi}$ is $H$-increasing. Thus $\varphi$ is $H$-increasing on $W_{0}:=\left\{X \in W: x \in \mathbb{R}_{+}^{n}\right\}$.

We have that

$$
\operatorname{Pg} \widetilde{M}_{j}=\left[\begin{array}{cc}
\alpha_{j} I & \operatorname{diag} \Delta\left(U_{1} M_{j} U_{2}^{T}\right) \\
\operatorname{diag} \Delta\left(U_{1} M_{j} U_{2}^{T}\right) & \alpha_{j} I
\end{array}\right]
$$

for any $g=g\left(U_{1}, U_{2}\right) \in G$. Using Corollary 2.3 and (2.9) with $g_{0}=h_{0}=U_{0}(\cdot) I$, one obtains

$$
\begin{aligned}
& \max _{1 \leq j \leq k} \varphi\left(\left[\begin{array}{cc}
\alpha_{j} I & \operatorname{diag} \Delta\left(U_{1} M_{j} U_{2}^{T}\right) U_{0} \\
\operatorname{diag} \Delta\left(U_{1} M_{j} U_{2}^{T}\right) U_{0} & \alpha_{j} I
\end{array}\right]\right) \\
& \quad \leq \varphi\left(\left[\begin{array}{cc}
\alpha_{0} I & \operatorname{diag} s\left(M_{0}\right) \\
\operatorname{diag} s\left(M_{0}\right) & \alpha_{0} I
\end{array}\right]\right),
\end{aligned}
$$

which is equivalent to (3.11). 
4. Concluding remarks. A central role in the proof of Theorem 2.1 is played by Schur type inequality (2.1) and by the double inequalities $P g w_{j} \preceq_{C} P g w \preceq_{H} w$ with the mediate vector $\mathrm{Pgw}$. Such kind of a relation is known in the literature as weak group majorization (see [8, p. 120]).

To be more precise, assume $\prec$ is a preordering on $W$ which is $H$-compactible, i.e., $\prec$ is invariant under finite convex combinations of elements of $H$ :

$$
y \prec x \text { implies } \sum_{i} \alpha_{i} h_{i} y \prec \sum_{i} \alpha_{i} h_{i} x
$$

for $h_{i} \in H$ and $\alpha_{i}>0$ with $\sum_{i} \alpha_{i}=1$. In particular, if $\prec$ is a cone preordering induced by an $H$-invariant convex cone, then $\prec$ is $H$-compactible (see [8, p. 120]). Given vectors $x, y \in V$, we say that $y$ is weakly $H$-majorized by $x$ (in symbol, $y \preceq_{H, w}$ $x$ ), if there exists $z \in W$ such that $y \prec z$ and $z \preceq_{H} x$.

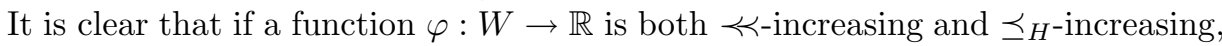
then $\varphi(y) \leq \varphi(x)$ whenever $y \preceq_{H, w} x$. In particular, this is valid if $\varphi$ is $\prec$-increasing, $H$-invariant and convex [8, Thm 3].

Summarizing, our results can be extended to an $H$-compactible ordering $\prec$ instead of the cone preordering $\preceq_{C}$, provided the linear operator $P g$ is $\prec$-isotone for each $g \in G$. Then Theorem 2.1 remains true for any $\preceq_{H, w}$-increasing function $\varphi: W \rightarrow \mathbb{R}$.

Also, further generalizations are possible for $H$-stochastic operators $L$ in place of $P g$. Recall that a linear operator $L: W \rightarrow W$ is said to be $H$-stochastic if $L x \preceq_{H} x$ for $x \in W$ (cf. [17, Thm 3.3]). Namely, if $w_{j} \prec w$ then $\varphi\left(L w_{j}\right) \leq \varphi(L w) \leq \varphi(w)$ provided $L$ is both $\prec$-isotone and $H$-doubly stochastic, and, in addition, $\varphi$ is both $\prec$-increasing and $H$-increasing. Moreover, $\varphi\left(L w_{j}\right) \leq \varphi(w)$ and $\max _{j} \varphi\left(L w_{j}\right) \leq \varphi(w)$ for any $\preceq_{H, w}$-increasing function $\varphi$.

\section{REFERENCES}

[1] S. A. Anderson and M. D. Perlman. Group-invariant analogues of Hadamard's inequality. Linear Algebra Appl., 110:91-116, 1988.

[2] A. Berman and R. J. Plemmons. A note on simultaneously diagonalizable matrices. Math. Inequal. Appl., 1(1):149-152, 1998.

[3] R. Bhatia. Matrix Analysis. Springer-Verlag, New York, 1997.

[4] M. L. Eaton. On group induced orderings, monotone functions, and convolution theorems. Inequalities in Statistics and Probability, Y. L. Tong, Editor, IMS Lectures NotesMonograph Series, 5:13-25, 1984.

[5] M. L. Eaton. Lectures on Topics in Probability Inequalities. CWI Tract 35, Centrum voor Wiskunde en Informatica, Amsterdam, 1987.

[6] M. L. Eaton. Group induced orderings with some applications in statistics. CWI Newsletter, 16:3-31, 1987.

[7] M. L. Eaton and M. D. Perlman. Reflection groups, generalized Schur functions, and the geometry of majorization. Ann. Probab., 5:829-860, 1977.

[8] A. Giovagnoli and H. P. Wynn. G-majorization with applications to matrix orderings. Linear Algebra Appl., 67:111-135, 1985.

[9] L. C. Grove and C. T. Benson. Finite Reflection Groups, Second edition. Springer - Verlag, New York, 1985.

[10] A. S. Lewis. Group invariance and convex matrix analysis. SIAM J. Matrix Anal. Appl., 17(4):927-949, 1996. 
[11] A. S. Lewis. Convex analysis on Cartan subspaces. Nonlinear Anal., 42:813-820, 2000.

[12] A. S. Lewis. Convex analysis on Hermitian matrices. SIAM J. Optim., 6(1):164-177, 1996.

[13] A. W. Marshall and I. Olkin. Inequalities: Theory of Majorization and Its Applications. Academic Press, New York, 1979.

[14] H. F. Miranda and R. C. Thompson. A trace inequality with a subtracted term. Linear Algebra Appl., 185:165-172, 1993.

[15] H. F. Miranda and R. C. Thompson. Group majorization, the convex hull of sets of matrices, and the diagonal elements-singular values inequalities. Linear Algebra Appl., 199:131-141, 1994.

[16] M. Niezgoda. Group majorization and Schur type inequalities. Linear Algebra Appl., 268:9-30, 1998.

[17] M. Niezgoda. G-majorization inequalities for linear maps. Linear Algebra Appl., 292:207-231, 1999.

[18] A. G. M. Steerneman. G-majorization, group-induced cone orderings and reflection groups. Linear Algebra Appl., 127:107-119, 1990.

[19] C. M. Theobald. An inequality for the trace of the product of two symmetric matrices. Math. Proc. Camb. Phil. Soc., 77:265-267, 1975. 